\title{
Repeatable Generalized Inverse Control Strategies for Kinematically Redundant Manipulators
}

\author{
Rodney G. Roberts Anthony A. Maciejewski \\ School of Electrical Engineering \\ Purdue University \\ West Lafayette, Indiana 47907
}

\begin{abstract}
A kinematically redundant manipulator possesses an infinite number of joint angle trajectories which satisfy a given desired end effector trajectory. The joint angle trajectories considered in this work are locally described by generalized inverses which satisfy the Jacobian equation relating the instantaneous joint angle velocities to the velocity of the end effector. One typically selects a solution from this set based on the local optimization of some desired physical property such as the minimization of the norm of the joint angle velocities, kinetic energy, etc. Unfortunately, this type of solution frequently does not possess the desirable property of repeatability in the sense that closed trajectories in the workspace are not necessarily mapped to closed trajectories in the joint space. In this work the issue of generating a repeatable control strategy which possess the desirable physical properties of a particular generalized inverse is addressed. This is done by first characterizing repeatable strategies using orthonormal basis functions to describe the null space of these transformations. The optimal repeatable inverse is then obtained by projecting the null space of the desired generalized inverse onto each of these basis functions. The resulting inverse is guaranteed to be the closest repeatable inverse to the desired inverse, in an integral norm sense, from the set of all inverses spanned by the selected basis functions. This technique is illustrated for a planar, three degree-offreedom manipulator.
\end{abstract}

\section{INTRODUCTION}

A robotic manipulator is described by its kinematic equation which relates the joint configurations of the manipulator to the position and orientation of the end effector in the workspace. The kinematic equation $\mathbf{f}: \Theta \rightarrow \mathcal{W}$ is usually a nonlinear mapping of the manipulators joint space $\theta$ to the workspace $\mathcal{W}$ where $\operatorname{dim}(\Theta)=n$ and $\operatorname{dim}(\mathcal{W})=m$. More specifically this equation is given by

$$
\mathbf{x}=\mathbf{f}(\theta)
$$

This work was supported in part by grants from the NEC Corporation and from TRW. where $X$ is an $m$-vector and $\theta$ is an $n$-vector. One of the popular techniques for controlling a manipulator is resolved motion rate control which calculates the joint velocities from the joint configuration and desired end effector velocity. The underlying equation is the Jacobian equation which for the positional component can be found by differentiating (1) to obtain

$$
\dot{\mathbf{x}}=J \dot{\theta}
$$

where $\dot{\mathbf{x}}$ is the desired end effector velocity. The chief advantage of using the Jacobian for the motion control of a manipulator is that the Jacobian is a linear relationship between the joint velocities and the end effector velocities. At each point $\theta, J$ is an $m \times n$ matrix.

Kinematically redundant manipulators are robotic systems which possess more degrees of freedom than are required for a specified task. This occurs when $m<n$. This additional freedom offers obvious advantages over conventional nonredundant manipulators including the potential for obstacle avoidance, torque minimization, singularity avoidance, and greater dexterity $[1,3,5,6,7,10,13,18,19,20]$. There are an infinite number of control strategies for redundant manipulators. One can take advantage of this freedom by choosing a control strategy which will optimize some particular criterion. A popular optimal control strategy is the minimum norm solution

$$
\dot{\theta}=J^{+} \dot{\mathbf{x}}
$$

where $J^{+}$is the Moore-Penrose pseudoinverse of $J$. This control strategy locally minimizes the joint velocities of the manipulator subject to moving the end effector along a specified trajectory. Since large joint velocities are associated with near-singular configurations, it was previously hoped that locally minimizing the joint velocities would avoid singularities; however this has been shown not to be true [2].

Equation (3) can be generalized to include all solutions by adding terms in the null space of $J$ which results in

$$
\dot{\theta}=J^{+} \dot{\mathbf{x}}+\left(I-J^{+} J\right) \mathbf{z}
$$

where $z$ is an arbitrary $n$-vector and $\left(I-J^{+} J\right) \mathrm{z}$ represents the orthogonal projection of $\mathrm{z}$ onto the null space of $J$. Liégeois [9] used $z$ to optimize a criterion function 
$g(\theta)$ subject to making the end effector follow a prescribed trajectory by setting $\mathrm{z}=\alpha \nabla g(\theta)$. This null space term has also been used for several other objectives including those listed previously $[6,10,13,18]$.

This work will consider generalized inverse strategies to solve (2) which are of the form

$$
\dot{\theta}=G \dot{\mathbf{x}}
$$

where $G$ satisfies $J G=I$ for nonsingular configurations. The elements of $G$ are functions of the joint configurations. This strategy may be chosen to locally minimize a given criterion function such as a least-squares minimum norm criteria on the joint velocities as in the case of the pseudoinverse solution. Also popular in the robotics literature are weighted pseudoinverse solutions which locally minimize $\dot{\theta}^{T} Q \dot{\theta}$ for some positive definite weighting matrix $Q$ such as the inertia matrix [17]. Due to the additional freedom afforded to kinematically redundant manipulators, control strategies such as (5) may not be repeatable in the sense that closed trajectories in the work space are not necessarily mapped to closed trajectories in the joint space so that for cyclic tasks the manipulator will not necessarily return to its starting configuration. Klein and Huang [8] give a proof of this for the pseudoinverse control of a three-link revolute manipulator. Such control strategies fail to give mappings which are one-to-one and onto.

Recently there has been significant interest in this issue of repeatability $[2,4,11,12,15,16]$. An elegant method for testing whether an inverse in the form (5) is repeatable for simply-connected, singularity-free subsets of the joint space was derived by Shamir and Yomdin [16]. This method, based on Frobenius's Theorem from differential geometry, consists of checking whether the Lie bracket of each pair of columns of $G$ lies in the column space of $G$. This straightforward but tedious calculation can be used to determine if the manipulator is repeatable for sufficiently small end effector movements. It can also be used to determine whether there exist what Shamir termed "stable surfaces" which are surfaces on which the control is repeatable for nonsingular configurations.

Research has also been done on the construction of repeatable control strategies. Mussa-Ivaldi and Hogan [12] have developed a class of repeatable inverses which use impedance control to devise strategies in the form of weighted-pseudoinverses. These inverses minimize criteria which lead to conservative solutions. Baillieul [2] devised a strategy which is repeatable in a simply-connected, singularity-free subset of the joint space. This method, called the extended Jacobian, minimizes a criterion function of the joint variables for certain initial conditions and will be discussed in greater detail in the following section. It is also possible to obtain repeatable controls by simply augmenting the Jacobian with the appropriate number of kinematic constraints [14].

The remainder of this paper is organized in the following manner: Section II describes a method for generating a generalized inverse $G$ from the null space of $G^{T}$. It is then shown how one can obtain repeatable inverses by suitably selecting the null space of $G^{T}$. Section III develops the mathematics required to define an appropriate class of augmenting vectors $\mathcal{V}$ which yield repeatable inverses. This class of augmenting vectors forms a closed subspace of a Hilbert space. Utilizing the mathematical structure on this set, Section IV discusses how to choose the augmenting vector in $\mathcal{V}$ which minimizes its distance from a set of null vectors of $G^{T}$. Finally Section V illustrates these concepts with an example.

\section{AUGMENTED JACOBIANS}

Any control strategy for a kinematically redundant manipulator can be found by inverting the square matrix obtained by augmenting the manipulator Jacobian with the appropriate row vectors. This is, of course, under the assumption that the square augmented matrix is nonsingular. Suppose that $J$ is augmented with a matrix $N^{T}$ so that

$$
J_{N}=\left[\begin{array}{c}
J \\
\cdots \\
N^{T}
\end{array}\right]
$$

The inverse of $J_{N}$, if it exists, has the form

$$
J_{N}^{-1}=\left[\begin{array}{ll}
G & M
\end{array}\right]
$$

where $G$ is a generalized inverse of $J$ and $M$ is a maximal rank matrix whose column space is exactly the null space of $J$. Setting $\mathbf{z}_{N}=N^{T} \dot{\theta}$ one obtains

$$
\left[\begin{array}{c}
\dot{\mathbf{x}} \\
\cdots \\
\mathbf{z}_{N}
\end{array}\right]=J_{N} \dot{\theta}
$$

so that

$$
\dot{\theta}=J_{N}^{-1}\left[\begin{array}{c}
\dot{\mathbf{x}} \\
\cdots \\
\mathrm{z}_{N}
\end{array}\right]=G \dot{\mathrm{x}}+M \mathrm{z}_{N} .
$$

In order to obtain the control in (5) one merely sets $\mathbf{z}_{N}=0$. In this work manipulators with one degree of redundancy are considered so that $N$ is a vector $\mathbf{v}$,

$$
J_{\mathbf{v}}=\left[\begin{array}{c}
J \\
\cdots \\
\mathbf{v}^{T}
\end{array}\right]
$$

and

$$
J_{v}^{-1}=\left[\begin{array}{ll}
G_{v} & \frac{n_{I}}{n_{J} \cdot \mathbf{v}}
\end{array}\right]
$$


where $n_{J}$ is any null vector of $J$. If for example one chose $\mathbf{v}$ to be proportional to $\mathbf{n}_{J}$ then $G_{\mathbf{v}}$ would be the pseudoinverse. One can consider the relationship of a generalized inverse and the set of its augmenting vectors as a one-to-one correspondence between the generalized inverses and an equivalence class on the augmenting vectors. This equivalence class on the augmenting vectors is described by the following equivalence relation: two augmenting vector functions $v$ and $w$ are equivalent on $\boldsymbol{\Omega}$ if there is a nowhere zero scalar function $\alpha$ such that $v=\alpha w$ almost everywhere on $\Omega$.

An example of an augmented Jacobian technique which guarantees repeatability in simply-connected subsets of the joint space that are free of singularities is the extend Jacobian method discussed earlier [2]. This method utilizes the redundancy to optimize a criterion function $g(\theta)$ along with the primary constraint of following a specified end effector trajectory. Suppose the manipulator starts at an optimal configuration $\theta^{*}$ for a given end effector position and orientation. Baillieul proved that a necessary condition for being at a local extremum is that the gradient of $g(\theta)$ possess no component along the null space, i.e.

$$
\nabla g\left(\theta^{*}\right) \cdot \mathbf{n}_{\mathrm{J}}\left(\theta^{*}\right)=0 .
$$

Combining the end effector constraint and the optimization criteria results in the equation

$$
\left[\begin{array}{c}
f(\theta) \\
\cdots \\
\nabla g \cdot \mathbf{n}_{\mathrm{J}}
\end{array}\right]=\left[\begin{array}{c}
\mathbf{x} \\
\cdots \\
\mathbf{0}
\end{array}\right] .
$$

Differentiating (13) results in

$$
\left[\begin{array}{c}
J \\
\cdots \\
\left(\nabla\left(\nabla g \cdot \mathbf{n}_{\mathrm{J}}\right)\right)^{T}
\end{array}\right] \dot{\theta}=\left[\begin{array}{c}
\dot{\mathbf{x}} \\
\cdots \\
0
\end{array}\right]
$$

where the matrix on the left-hand side is defined as the extended Jacobian, denoted $J_{e}$ [2]. If $J_{e}$ is nonsingular then one obtains the joint velocities by simply multiplying (14) by $J_{e}^{-1}$. Clearly, $J_{e}$ will be singular at kinematic singularities of the original robot which correspond to the singularities of $J$. However, $J_{e}$ may also become singular when any of the additional rows added to the Jacobian are a linear combination of the rows of $J$. Mathematically, these singularities can be identified by evaluating the equation

$$
\mathbf{n}_{\mathbf{J}} \cdot \nabla\left(\nabla \boldsymbol{g} \cdot \mathbf{n}_{\mathbf{J}}\right)=0
$$

These types of singularities, which are typical of augmented Jacobians, were noted by Baillieul for which he coined the term "algorithmic singularities" [2].

The repeatable inverses can be found by inverting $J_{v}$ where $v$ is a gradient. By augmenting the Jacobian with a gradient one is resolving the manipulator's redundancy by adding to the kinematic equation an additional function $h$ where $v=\nabla h$. By adding this additional function the manipulator acts "mathematically" like a nonredundant manipulator assuming that the rows of $J$ and $v$ are linearly independent. In fact it is these gradients which define the class of control strategies which are repeatable in simply-connected, singularity-free domains. Later a set of allowable augmenting vectors which result in repeatable control strategies will be defined. The elements of this set will consist of gradient functions. It is important to note that this technique is distinct from the extended Jacobian technique since there may be no function $g$ which describes the desired optimization criterion. The proposed technique is able to handle more general optimization criteria which are not restricted to be only functions of $\theta$. In particular, one can consider the minimum joint velocity norm solution obtained using the pseudoinverse, which will be used as an illustrative example in the remainder of this work. The same technique can be used for any other desirable generalized inverse $G$ by substituting the null vectors of $G^{T}$.

\section{MATHEMATICAL PRELIMINARIES}

In order to pursue this characterization of repeatable control strategies, this section will present the mathematical machinery needed to properly define an appropriate class of augmenting vectors which result in repeatable control strategies. The spaces that will be considered in this work are subspaces of the separable Hilbert space $\mathcal{L}_{2}(\Omega)$, the space of Lebesgue measurable functions $\mathbf{u}: \Omega \rightarrow \mathbb{R}^{n}$ which satisfy $\int_{\Omega}\|\mathbf{u}\|_{2}^{2} d \theta<\infty$. An important property of any Hilbert space is that it possesses an inner product. The inner product $\langle\cdot, \cdot\rangle_{\Omega}$ on two vector functions $u, v$ in $\mathcal{L}_{2}(\Omega)$ is defined to be

$$
<\mathbf{u}, \mathbf{v}>_{\Omega}=\int_{\Omega} \mathbf{u} \cdot \mathbf{v} d \theta
$$

where $u \cdot v$ is the standard dot product and $\int_{\Omega} d \theta$ is the Lebesgue integral on $\Omega \subset \theta$. The corresponding integral norm

$$
\|\mathbf{u}\|_{\Omega}=\left[\int_{\Omega}\|\mathbf{u}\|_{2}^{2} d \theta\right]^{\frac{1}{2}}
$$

will be used as a measure of the distance between vector functions on $\Omega$. Since $\mathcal{L}_{2}(\Omega)$ is a Hilbert space it follows that for any closed subspace $U$ and any $w \in \mathcal{L}_{2}(\Omega)$ there exists a unique $u \in U$ such that $\|\mathbf{u}-\mathbf{w}\|_{\Omega}$ is minimal.

While $\|\mathbf{u}-\mathbf{w}\|_{\Omega}$ gives a measure of the distance between two elements $u$ and $w$ in $\mathcal{L}_{2}(\Omega)$, the measure of the distance between two subsets $F$ and $G$ of $\mathcal{L}_{2}(\Omega)$ is defined to be $\operatorname{dist}(F, G)=\inf \left\{\|\mathbf{u}-\mathbf{v}\|_{\Omega} \mid \mathbf{u} \in F, \mathbf{v} \in G\right\}$. In particular this work is concerned with the distance between a 
subset $\mathcal{N}_{0}$ of the null space $\mathcal{N}$ of $J$ and a space of allowable augmenting vectors $\mathcal{V}$. This subset $\mathcal{N}_{0}$ is the set of null vector functions which are normalized in the norm $\|\cdot\| \Omega$. A desirable gradient $\mathbf{v}^{*}$ will have the property that it minimizes inf $_{n \in \mathcal{N}_{0}}\left\|v^{*}-\mathbf{n}\right\|_{\Omega}$. Clearly for any vector function $\mathbf{n} \in \mathcal{N}_{\mathbf{0}}$ the element in $\mathcal{V}$ which minimizes $\|\mathbf{v}-\mathbf{n}\|_{\mathbf{\Omega}}$ is exactly the orthogonal projection of $\mathbf{n}$ onto $\mathcal{V}$. For the space $\mathcal{N}_{0}$ each element can be written in the form $\alpha \hat{\mathbf{n}}_{J}$ for nonsingular configurations where $\alpha$ is any continuous function and $\left\|\hat{\mathbf{n}}_{J}\right\|_{2}=1$. Thus if $\Omega$ does not contain any singularities, $\mathcal{N}_{0}(\Omega)=\left\{\alpha \hat{\mathbf{n}}_{J} \mid \int_{\Omega} \alpha^{2} d \theta=1\right\}$. A rigorous definition can be made of an allowable space of augmenting vectors which will define the repeatable control strategies of interest. Consider the space $\mathcal{V}_{1}=\left\{\nabla g \in \mathcal{L}_{2}(\Omega) \mid g \in C^{1}(\Omega)\right\}$. An allowable space of augmenting vectors $\mathcal{V}$ is defined to be any closed linear subspace of $\mathcal{V}_{1}$ which has an orthonormal basis $\left\{v_{i}\right\}_{i \geq 1}$. The property that the subspace is closed is important since this guarantees that for any vector function $u$ in $\mathcal{L}_{2}(\Omega)$ there is an element in the subspace $\mathcal{V}$ which is closest to $\mathbf{u}$.

An example of such a subspace is the span of any finite orthonormal subset of $\mathcal{V}_{1}$. Section $V$ illustrates how one might go about choosing such an orthonormal basis for a manipulator with revolute joints. With these restrictions on $\mathcal{V}$ the Projection Theorem guarantees that the element of $\mathcal{V}$ which is closest to some arbitrary vector function $\mathbf{n}$ in $\mathcal{L}_{2}(\Omega)$ is given by

$$
\mathbf{v}^{*}(\mathbf{n})=\sum_{i \geq 1}<\mathbf{n}, \mathbf{v}_{i}>_{\mathbf{n}} \mathbf{v}_{i}
$$

which is simply the orthogonal projection of $\mathbf{n}$ onto $\mathcal{V}$.

\section{A CLASS OF OPTIMAL REPEATABLE INVERSES}

In order to do actual calculations and an implementation one is forced to consider a finite-dimensional subspace of $\mathcal{V}$ of dimension say $N$. Let this subspace be denoted by $\mathcal{V}_{N}$ and let $\left\{\mathbf{v}_{j}\right\}_{j=1}^{N}$ be an orthonormal basis for $\mathcal{V}_{N}$. Now that an appropriate subspace has been defined, one can choose an augmenting vector from this set which minimizes its distance from $\mathcal{N}_{0}$, the space of null vectors which are of unit length in the norm $\|\cdot\|_{\Omega}$. This is done by finding the $\alpha^{*}$ in $\mathcal{A}$ and the $v$ in $\mathcal{V}_{N}$ which minimizes $\left\|\alpha^{*} \hat{\mathbf{n}}_{J}-\mathbf{v}\right\|_{\Omega}^{2}$. This minimization will be done in two steps. First the form of the $\alpha$ 's which are closest to $V_{N}$ will be derived. Then the corresponding $\mathbf{v}$ in $\mathcal{V}_{N}$ for each of the candidate $\alpha$ 's is calculated. Finally the minimal pair is chosen from these candidates.

The $\mathbf{n}$ in $\mathcal{N}_{0}$ which is closest to a $\mathbf{v}$ in $\mathcal{V}_{N}$ is characterized in terms of its corresponding $\alpha$ by the following proposition:

Proposition 1 Let $v=\sum_{i=1}^{N} c_{i} v_{i}$ be a fixed vector function in $\mathcal{V}_{N}$ and suppose that $\sum_{i=1}^{N} c_{i} \mathbf{v} \cdot \hat{\mathbf{n}}_{J} \neq 0$. Suppose $\alpha^{*}=\arg \min _{\alpha \in \mathcal{A}}\left\|\alpha \hat{\mathbf{n}}_{J}-\sum_{i=1}^{N} c_{i} v_{i}\right\|_{\Omega}^{2}$. Then there exists a constant $K$ such that

$$
\alpha^{*}=K \sum_{i=1}^{N} c_{i} \mathbf{v}_{i} \cdot \hat{\mathbf{n}}_{J}
$$

\section{Proof See Appendix A.}

Thus the candidate $\alpha$ 's are of the form given in (19). It is thus only necessary to consider $\hat{\mathcal{A}}$, the set of functions in $\mathcal{A}$ which have this form.

For each $\mathrm{n} \in \mathcal{N}_{0}$ the corresponding $\mathbf{v} \in \mathcal{V}_{N}$ which is closest to $\mathbf{n}$ is the orthogonal projection of $\mathbf{n}$ onto $\mathcal{V}$. Let $\alpha$ be in $\hat{\mathcal{A}}$ and let $\mathbf{v}(\alpha)$ denote the orthogonal projection of $\alpha \hat{\mathbf{n}}_{J}$ onto $\mathcal{V}_{N}$. Thus the problem now becomes to minimize $\left\|\alpha \hat{\mathbf{n}}_{J}-\mathbf{v}(\alpha)\right\|_{\Omega}$ over the scalar functions $\alpha$ in $\hat{\mathcal{A}}$. Since $\alpha$ is in $\hat{\mathcal{A}}$, there exist $b_{1}, \ldots, b_{N}$ such that

$$
\alpha=\sum_{j=1}^{N} b_{j} \mathbf{v}_{j} \cdot \hat{\mathbf{n}}_{J}
$$

Let $a_{i}(\alpha)$ be the generalized Fourier coefficient of $\alpha \hat{\mathbf{n}}_{J}$ corresponding to $v_{i}$. From (20) it follows that

$$
a_{i}(\alpha)=\left\langle\alpha \hat{\mathbf{n}}_{J}, \mathbf{v}_{i}>_{\Omega}=\sum_{j=1}^{N} b_{j} \int_{\Omega}\left(\mathbf{v}_{i} \cdot \hat{\mathbf{n}}_{J}\right)\left(\mathbf{v}_{j} \cdot \hat{\mathbf{n}}_{J}\right) d \theta\right.
$$

In order to make the presentation clearer some vector notation is introduced. Let $\mathbf{a}=\left[a_{1}(\alpha), \ldots, a_{N}(\alpha)\right]^{T}$ correspond to the series $\sum_{j=1}^{N} a_{j} \mathbf{v}_{j}, \mathbf{b}=\left[b_{1}, \ldots, b_{N}\right]$, and let the matrix $M$ be given by

$$
M_{i j}=\int_{\mathbf{\Omega}}\left(\mathbf{v}_{i} \cdot \hat{\mathbf{n}}_{J}\right)\left(\mathbf{v}_{j} \cdot \hat{\mathbf{n}}_{J}\right) d \theta
$$

The matrix $M$ is the Gramian matrix. Using this notation (21) becomes

$$
\mathbf{a}=M \mathbf{b} .
$$

In order for $\left\|\alpha \hat{\mathbf{n}}_{J}\right\|_{\Omega}=1$ there is a restriction on $b$. Integrating the square of $(20)$ yields

$$
\int_{\Omega} \alpha^{2} d \theta=\sum_{i=1}^{N} \sum_{j=1}^{N} \int_{\Omega}\left(\mathbf{v}_{i} \cdot \hat{\mathbf{n}}_{J}\right)\left(\mathbf{v}_{j} \cdot \hat{\mathbf{n}}_{J}\right) d \theta b_{i} b_{j}
$$

which is equal to one, which in vector notation becomes

$$
\mathbf{b}^{T} M \mathbf{b}=1 \text {. }
$$

Now $\left\|\alpha \hat{\mathbf{n}}_{J}-\mathbf{v}(\alpha)\right\|_{\Omega}^{2}=1-\mathbf{a}^{T} \mathbf{a}$. Thus it is important to maximize $\mathbf{a}^{T}$ a subject to (25). This maximum occurs when $\mathbf{a}$ and $\mathbf{b}$ are eigenvectors of $M$ associated with its 
largest eigenvalue. Since $M$ is a symmetric positive semidefinite matrix, a can be found from the singular value decomposition of $M$. The vector a would simply be $\sqrt{\sigma_{1}} \mathbf{u}_{1}$ where $\sigma_{1}$ is the largest singular value and $u_{1}$ is its corresponding singular vector. It is this vector of generalized Fourier coefficients which minimizes the distance to $\mathcal{N}_{0}$.

\section{EXAMPLE}

In order to illustrate the previous section consider a planar 3R manipulator which has links of unit length. The Jacobian for this particular manipulator is

$J=\left[\begin{array}{ccc}-s \theta_{1}-\mathrm{s} \theta_{12}-\mathrm{s} \theta_{123} & -\mathrm{s} \theta_{12}-\mathrm{s} \theta_{123} & -\mathrm{s} \theta_{123} \\ \mathrm{c} \theta_{1}+\mathrm{c} \theta_{12}+\mathrm{c} \theta_{123} & \mathrm{c} \theta_{12}+\mathrm{c} \theta_{123} & \mathrm{c} \theta_{123}\end{array}\right]$

where $\theta_{i j}=\theta_{i}+\theta_{j}$. For a simply-connected, singularityfree subset of the joint space the unit null vector $\hat{\mathbf{n}}_{J}$ for this manipulator can be continuously and uniquely defined up to a multiple of -1 . In particular it can be obtained by normalizing the cross product of the two rows of the Jacobian:

$$
\hat{\mathbf{n}}_{J}=\frac{1}{\Delta}\left[\begin{array}{c}
\mathrm{s} \theta_{3} \\
-\mathrm{s} \theta_{3}-\mathrm{s} \theta_{23} \\
\mathrm{~s} \theta_{2}+\mathrm{s} \theta_{23}
\end{array}\right]
$$

where $\Delta=\sqrt{s^{2} \theta_{3}+\left(\mathrm{s} \theta_{3}+\mathrm{s} \theta_{23}\right)^{2}+\left(\mathrm{s} \theta_{2}+\mathrm{s} \theta_{23}\right)^{2}}$. For this example the desired optimization criterion will be to minimize the norm of the joint angle velocities. The exact solution for this criterion is given by the pseudoinverse of the Jacobian, however, it is well-known that the pseudoinverse is not repeatable. Since the class of augmenting vectors associated with the pseudoinverse is characterized by the null vectors of the Jacobian, the task at hand is to find a gradient augmenting vector that most closely matches a null vector of the Jacobian in a simplyconnected, singularity-free region $\Omega=I_{1} \times I_{2} \times I_{3}$ where $I_{i}=\left[a_{i}, b_{i}\right]$ with $a_{i}<b_{i}$ for $i=1,2,3$. The boundaries of this region can be chosen based on the particular physical constraints of the manipulator or the requirements of the task being performed.

The first step is to determine an orthonormal basis for the allowable augmenting vectors. The Fourier functions for each interval $I_{i}$ are given by

$$
\left\{\frac{K_{i}}{\sqrt{2}}, K_{i} \sin \frac{2 n \pi}{\left|I_{i}\right|}\left(\theta_{i}-c_{i}\right), K_{i} \cos \frac{2 n \pi}{\left|I_{i}\right|}\left(\theta_{i}-c_{i}\right)\right\}_{n \geq 1}
$$

where $\left|I_{i}\right|=b_{i}-a_{i}, c_{i}=\frac{1}{2}\left(a_{i}+b_{i}\right)$ and $K_{i}=\sqrt{2 /\left|I_{i}\right|}$. The Fourier functions on $\Omega$, denoted here by $\rho_{j}$, are simply permutations of the products of the Fourier functions for each $I_{i}$ described by the set given in (28). The set $\left\{\rho_{j}\right\}_{j \geq 1}$ forms a basis for the scalar functions on $\Omega$. By taking the gradient of each $\rho_{j}$ one can obtain a basis for a subset of gradients. It is important to note that this basis does not span the entire space of gradient functions. However, the set $\left\{\nabla \rho_{j}\right\}_{j \geq 1}$ does form an orthogonal basis for a proper subset of the gradients (see Appendix B), which is crucial for the success of this technique. Reducing this set to $N$ terms and normalizing each element in the norm $\|\cdot\|_{\Omega}$, results in the space $\mathcal{V}_{N}=\operatorname{span}\left\{\nabla \rho_{j}\right\}_{j=1}^{N}$ which forms a closed subspace of $\mathcal{L}_{2}(\Omega)$ where each member is a gradient function. For this example, $\Omega$ will be taken to be $[\pi / 4,3 \pi / 4]^{3}$. Thus $(28)$ becomes

$$
\left\{\sqrt{\frac{2}{\pi}}, \frac{2}{\sqrt{\pi}} \sin 4 n \theta_{i}, \frac{2}{\sqrt{\pi}} \cos 4 n \theta_{i}\right\}_{n \geq 1} .
$$

For the purposes of illustration, the following orthonormal set of six vector functions will be used

$$
\begin{gathered}
\left\{\kappa\left[\begin{array}{c}
\cos 4 \theta_{1} \\
0 \\
0
\end{array}\right], \kappa\left[\begin{array}{c}
0 \\
\cos 4 \theta_{2} \\
0
\end{array}\right], \kappa\left[\begin{array}{c}
0 \\
0 \\
\cos 4 \theta_{3}
\end{array}\right],\right. \\
\left.\kappa\left[\begin{array}{c}
\sin 4 \theta_{1} \\
0 \\
0
\end{array}\right], \kappa\left[\begin{array}{c}
0 \\
\sin 4 \theta_{2} \\
0
\end{array}\right], \kappa\left[\begin{array}{c}
0 \\
0 \\
\sin 4 \theta_{3}
\end{array}\right]\right\}
\end{gathered}
$$

where $\kappa=4 / \pi^{3 / 2}$. Let $\mathcal{V}_{6}$ denote the span of the above set. It can be easily verified that this is an orthonormal subset of $\mathcal{V}$.

The fact that $\mathcal{V}_{6}$ is closed guarantees that there is an element in $\mathcal{V}_{6}$ which is closest to some $\mathbf{n}_{J}$ in $\mathcal{N}_{0}$. Thus from the Projection Theorem it follows that for each $\mathbf{n}_{J} \in \mathcal{N}_{0}$ the unique $\mathbf{v}^{*} \in \mathcal{V}_{6}$ which minimizes its distance from $n_{J}$ is given by

$$
\mathbf{v}^{*}=\sum_{j=1}^{6}\left\langle\mathbf{n}_{J}, \mathbf{v}_{j}>_{\Omega} \mathbf{v}_{j}\right.
$$

where $\mathbf{v}_{j}=\nabla \rho_{j} /\left\|\nabla \rho_{j}\right\|_{\Omega}$.

The Gramian matrix corresponding to $\mathcal{V}_{6}$ is calculated using (22) and is given below:

$$
M=\left[\begin{array}{cccccc}
.428 & 0 & 0 & 0 & 0 & 0 \\
0 & .285 & -.021 & 0 & -.026 & .011 \\
0 & -.021 & .292 & 0 & .009 & .026 \\
0 & 0 & 0 & .428 & 0 & 0 \\
0 & -.026 & .009 & 0 & .284 & .029 \\
0 & .011 & .026 & 0 & .029 & .285
\end{array}\right] .
$$

Since $M$ is symmetric it can be decomposed into the form $U S U^{T}$ where $U$ is a unitary matrix and $S$ is a diagonal matrix. For this case

$$
U=\left[\begin{array}{cccccc}
1 & 0 & 0 & 0 & 0 & 0 \\
0 & 0 & -.394 & .756 & .005 & -.523 \\
0 & 0 & .558 & .040 & .749 & -.355 \\
0 & 1 & 0 & 0 & 0 & 0 \\
0 & 0 & .546 & -.073 & -.650 & -.523 \\
0 & 0 & .486 & .649 & -.125 & .572
\end{array}\right]
$$


and

$$
S=\left[\begin{array}{cccccc}
.428 & 0 & 0 & 0 & 0 & 0 \\
0 & .428 & 0 & 0 & 0 & 0 \\
0 & 0 & .338 & 0 & 0 & 0 \\
0 & 0 & 0 & .296 & 0 & 0 \\
0 & 0 & 0 & 0 & .279 & 0 \\
0 & 0 & 0 & 0 & 0 & .233
\end{array}\right] .
$$

The largest eigenvalue of $M$ is 0.428 . The two corresponding vectors are $\left[\begin{array}{llllll}1 & 0 & 0 & 0 & 0 & 0\end{array}\right]^{T}$ and $\left[\begin{array}{llllll}0 & 0 & 0 & 1 & 0 & 0\end{array}\right]^{T}$. Thus $\sqrt{0.428} \mathrm{v}_{1}$ and $\sqrt{0.428} \mathrm{v}_{4}$ are vector functions in $\mathcal{V}_{6}$ which have minimum distance from $\mathcal{N}_{0}$. In fact any linear combination of $\mathbf{v}_{1}$ and $\mathbf{v}_{4}$ which has euclidean length $\sqrt{0.428}$ is of minimum distance from $\mathcal{N}_{0}$ over the space $\mathcal{V}_{6}$. Therefore, a practical choice for the augmented Jacobian becomes

$J_{\mathbf{v}}=\left[\begin{array}{ccc}-\mathrm{s} \theta_{1}-\mathrm{s} \theta_{12}-\mathrm{s} \theta_{123} & -\mathrm{s} \theta_{12}-\mathrm{s} \theta_{123} & -\mathrm{s} \theta_{123} \\ \mathrm{c} \theta_{1}+\mathrm{c} \theta_{12}+\mathrm{c} \theta_{123} & \mathrm{c} \theta_{12}+\mathrm{c} \theta_{123} & \mathrm{c} \theta_{123} \\ \frac{4 \sqrt{0.428}}{\mathrm{~s} / 2} \cos 4 \theta_{1} & 0 & 0\end{array}\right]$

which results in a repeatable inverse that approximates the pseudoinverse.

\section{CONCLUSION}

The contribution of this work is a technique for generating a repeatable generalized inverse which is close to some arbitrary generalized inverse that possesses desirable properties. This technique relies on using orthonormal basis functions to describe a set of possible gradient functions. While this is in general a formidable task, it was shown that for this particular application, simple trigonometric functions are an ideal choice. It was also shown that the optimal coefficients for these basis functions can be easily determined by calculating the singular vector associated with the maximum singular value of the Gramian matrix. Finally, an example was presented which illustrated this technique.

\section{APPENDIX A}

Proposition 1 Let $\mathbf{v}=\sum_{i=1}^{N} c_{i} \mathbf{v}_{i}$ be a fixed vector function in $\mathcal{V}_{N}$ and suppose that $\sum_{i=1}^{N} c_{i} \mathbf{v} \cdot \hat{\mathbf{n}}_{J} \neq 0$. Suppose $\alpha^{*}=\arg \min _{\alpha \in \mathcal{A}}\left\|\alpha \hat{\mathbf{n}}_{J}-\sum_{i=1}^{N} c_{i} \mathbf{v}_{i}\right\|_{\Omega}^{2}$. Then there exists a constant $K$ such that

$$
\alpha^{*}=K \sum_{i=1}^{N} c_{i} \mathbf{v}_{i} \cdot \hat{\mathbf{n}}_{J}
$$

Proof The function $\alpha^{*}$ solves the following problem:

$$
\begin{array}{ll}
\text { Minimize } & \int_{\Omega}\left\|\alpha \hat{\mathbf{n}}_{J}-\sum_{i=1}^{N} c_{i} \mathbf{v}_{i}\right\|_{2}^{2} d \theta \\
\text { Subject to } & \int_{\Omega} \alpha^{2} d \theta=1 .
\end{array}
$$

The Euler-Lagrange equation must be satisfied in order for a minimum to occur. This equation is given by

$$
\frac{\partial}{\partial \alpha}\left\|\alpha \hat{\mathbf{n}}_{J}-\sum_{i=1}^{N} c_{i} \mathbf{v}_{i}\right\|_{2}^{2}+\lambda \frac{\partial}{\partial \alpha} \alpha^{2}=0
$$

which becomes

$$
2 \alpha-2 \sum_{i=1}^{N} c_{i} \mathbf{v}_{i} \cdot \hat{\mathbf{n}}_{J}+2 \lambda \alpha=0
$$

Separating terms results in

$$
(1+\lambda) \alpha=\sum_{i=1}^{N} c_{i} \mathbf{v}_{i} \cdot \hat{\mathbf{n}}_{J}
$$

Since $\sum_{i=1}^{N} c_{i} \mathbf{v}_{i} \cdot \hat{\mathbf{n}}_{J} \neq 0$, one obtains

$$
\alpha=\frac{1}{1+\lambda} \sum_{i=1}^{N} c_{i} \mathbf{v}_{i} \cdot \hat{\mathbf{n}}_{J}
$$

By setting $K=\frac{1}{1+\lambda}$ the lemma is proved. Note that $K$ can be calculated from the fact that $\alpha$ is of unit length in the norm $\|\cdot\| \Omega$.

\section{APPENDIX B}

Proposition $2\left\{\frac{\nabla \rho_{j}}{\left\|\nabla \rho_{j}\right\| \Omega}\right\}_{j=1}^{N}$ is an orthonormal set.

Proof In order to prove the proposition it suffices to show that any two distinct elements of the set are orthogonal. Let $j \neq k$ so that $\rho_{j} \neq \rho_{k}$. Both have the form of the product of trigonometric functions

$$
\begin{gathered}
\rho_{j}=R_{1 m_{1}}\left(\theta_{1}\right) R_{2 m_{2}}\left(\theta_{2}\right) R_{3 m_{3}}\left(\theta_{3}\right) \\
\rho_{k}=T_{1 p_{1}}\left(\theta_{1}\right) T_{2 p_{2}}\left(\theta_{2}\right) T_{3 p_{3}}\left(\theta_{3}\right)
\end{gathered}
$$

where $R_{i n}$ and $T_{i n}$ are Fourier functions on $I_{i}$ of the form (28). The gradients are

$$
\nabla \rho_{j}=\left[\begin{array}{l}
R_{1 m_{1}}^{\prime}\left(\theta_{1}\right) R_{2 m_{2}}\left(\theta_{2}\right) R_{3 m_{3}}\left(\theta_{3}\right) \\
R_{1 m_{1}}\left(\theta_{1}\right) R_{2 m_{2}}^{\prime}\left(\theta_{2}\right) R_{3 m_{3}}\left(\theta_{3}\right) \\
R_{1 m_{1}}\left(\theta_{1}\right) R_{2 m_{2}}\left(\theta_{2}\right) R_{3 m_{3}}^{\prime}\left(\theta_{3}\right)
\end{array}\right]
$$

and

$$
\nabla \rho_{k}=\left[\begin{array}{l}
T_{1 p 1}^{\prime}\left(\theta_{1}\right) T_{2 p_{2}}\left(\theta_{2}\right) T_{3 p_{3}}\left(\theta_{3}\right) \\
T_{1 p 1}\left(\theta_{1}\right) T_{2 p_{3}}^{\prime}\left(\theta_{2}\right) T_{3 p_{3}}\left(\theta_{3}\right) \\
T_{1 p 1}\left(\theta_{1}\right) T_{2 p_{2}}\left(\theta_{2}\right) T_{3 p_{3}}^{\prime}\left(\theta_{3}\right)
\end{array}\right]
$$


where $R_{i m_{i}}^{\prime}$ and $T_{i p_{i}}^{\prime}$ represent the derivative with respect to $\theta_{i}$ of $R_{i m_{i}}$ and $T_{i p_{i}}$, respectively. The inner product of $\nabla \rho_{j}$ and $\nabla \rho_{k}$ is

$$
\begin{aligned}
\int_{\Omega} \nabla \rho_{j} \cdot \nabla \rho_{k} d \theta & =\int_{\Omega} \sum_{i=1}^{3}\left(R_{i m_{i}}^{\prime} \prod_{l \neq i} R_{l m_{l}}\right)\left(T_{i p_{i}}^{\prime} \prod_{l \neq i} T_{l p_{l}}\right) d \theta \\
& =\sum_{i=1}^{3} \int_{\Omega} R_{i m_{i}}^{\prime} T_{i p_{i}}^{\prime} \prod_{l \neq i} R_{l m_{l}} T_{l p_{l}} d \theta \\
& =\sum_{i=1}^{3} \int_{I_{i}} R_{i m_{i}}^{\prime} T_{i p_{i}}^{\prime} d \theta_{i} \prod_{l \neq i} \int_{I_{l}} R_{l m_{l}} T_{l p_{l}} d \theta_{l}
\end{aligned}
$$

Since $\rho_{j} \neq \rho_{k}$ there is an $i$ such that $R_{i m_{i}} \neq T_{i p_{i}}$. Then $R_{i m_{i}}$ and $T_{i p_{i}}$ are orthogonal. Since both are of the form (28), $R_{i m_{i}}^{\prime}$ and $T_{i p_{i}}^{\prime}$ are also orthogonal. It thus follows that

$$
\int_{\Omega} \nabla \rho_{i} \cdot \nabla \rho_{j} d \theta=0
$$

$Q E D$

\section{REFERENCES}

[1] J. Baillieul, J. Hollerbach, and R. Brockett, "Programming and control of kinematically redundant manipulators," in Proc. 24th IEEE Conf. Decision Control, Las Vegas, Dec. 12-14, 1984, pp. 768-774.

[2] J. Baillieul, "Kinematic programming alternatives for redundant manipulators," in Proc. 1985 IEEE Int. Conf. Robotics Automat., St. Louis, MO, March 1985, pp. 722-728.

[3] J. Baillieul, "Avoiding obstacles and resolving redundancy," in Proc. 1986 IEEE Int. Conf. Robotics Automat., San Francisco, CA, April 1986, pp. 16981703.

[4] D. R. Baker and C. W. Wampler II, "On the inverse kinematics of redundant manipulators", Int. J. Robotics Res., vol. 7, no. 2, pp. 3-21, March/April 1988.

[5] R. Dubey and J. Y.S. Luh, "Redundant robot control for higher flexibility," in Proc. 1987 IEEE Int. Conf. Robotics Automat., Raleigh, N.C., March-April 1987, pp. 1066-1072.

[6] J. M. Hollerbach and K. C. Suh, "Redundancy resolution of manipulators through torque optimization," IEEE J. Robotics Automat., vol. RA-3, no. 4, pp. 308316, Aug. 1987.

[7] C. A. Klein and B. E. Blaho, "Dexterity measures for the design and control of kinematically redundant manipulators," Int. J. Robotics Res., vol. 6, no. 2 , pp. 72-83, Su 1987.
[8] C. A. Klein and C. H. Huang, "Review of pseudoinverse control for use with kinematically redundant manipulators," IEEE Trans. Sys., Man Cyber., vol. SMC-13, no. 3, pp. 245-250, March/April 1983.

[9] A. Liégeois, "Automatic supervisory control of the configuration and behavior of multibody mechanisms," IEEE Trans. Sys., Man Cyber., vol.SMC-12, no. 12, pp. 868-871, Dec. 1977.

[10] A. A. Maciejewski and C. A. Klein, "Obstacle avoidance for kinematically redundant manipulators in dynamically varying environments," Int. J. Robotics Res., vol. 4, no. 3, pp. 109-117, Fall 1985.

[11] A. A. Maciejewski and R. G. Roberts, "Utilizing kinematic redundancy in robotic systems: Practical implementations and fundamental limitations," in Proc. $A C C$, San Diego, CA, May 23-25, 1990, pp. 209-214.

[12] F. A. Mussa-Ivaldi and N. Hogan, "Solving kinematic redundancy with impedance control: a class of integrable pseudoinverses," in Proc. 1985 IEEE Int. Conf. Robotics Automat., Scottsdale, AZ, 1989, pp. 283-288.

[13] Y. Nakamura, H. Hanafusa, and T. Yoshikawa, "Task-priority based redundancy control of robot manipulators," Int. J. Robotics Res., vol. 6, no. 2, pp. 315, Su 1987.

[14] H. Seraji, "Configuration control of redundant manipulators: Theory and implementation," IEEE Trans. Robotics Automat., vol. 5, no. 4, pp. 472-490, Aug. 1989.

[15] T. Shamir, "Remarks on some dynamical problems of controlling redundant manipulators," IEEE Trans. Automatic Control, vol. 35, no. 3, pp. 341-344, March 1990.

[16] T. Shamir and Y. Yomdin, "Repeatability of redundant manipulators: Mathematical solution of the problem," IEEE Trans. Automatic Control, vol. 33, no. 11, pp. 1004-1009, Nov. 1988.

[17] D. E. Whitney, "Resolved motion rate control of manipulators and human prostheses," IEEE Trans. Man-Machine Syst., vol. MMS-10, pp. 47-53, June 1969.

[18] T. Yoshikawa, "Analysis and control of robot manipulators with redundancy," in Robotics Research: The First International Symposium, M. Brady and R. Paul, Eds., pp. 735-747, MIT Press, 1984.

[19] T. Yoshikawa, "Manipulability of robotic mechanisms," Int. J. Robotics Res., vol. 4, no. 2, pp. 3-9, Su 1985.

[20] T. Yoshikawa, "Dynamic manipulability of robot manipulators," J. Robotic Sys., vol. 2, no. 1, pp. 113-124, 1985. 